\title{
Restoration of malperfusion during repair of thoracic aortic dissection
}

\author{
Jeffrey Shuhaiber ${ }^{1}$ and Volodymyr Labinskyy ${ }^{2}$ \\ ${ }^{1}$ Baystate Medical Center \\ ${ }^{2}$ Affiliation not available
}

April 15, 2021

\begin{abstract}
A 46- year old male, during intense physical activity, sustained malperfusion of the lower extremities from Type A thoracic aortic dissection. We took him to the operating room. emergently and perfusing both lower extremities using a modified straight graft with side to side anastomosis to one limb and end to side anastomoses to the inflow perfusion and contralateral limb. The patient was successfully discharged and remained to be doing well. for four years.
\end{abstract}

\section{Introduction}

Malperfusion syndrome in acute aortic dissection is associated with higher post repair

Hospital mortality. We describe a simple and facile technique to restore extremity malperfusion at the time of aortic dissection repair.

\section{Case Report}

A 46-year old otherwise healthy individual man experienced sudden onset of severe

thoracolumbar pain with radiation to both lower extremities during climactic sexual intercourse.

The IRB waived the right for any formal review. He noticed weakness and rigidity in both lower extremities that he initially attributed to spasm.

There was no family of aortic aneurysm or dissection. Furthermore, when he arrived at our emergency room, he was in agonizing lower extremity pain refractory to pain medications. A computed tomography angiogram (CTA) confirmed a type A aortic dissection extending from the aortic root to the right common femoral artery (CFA). The dissection flap extended superiorly into bilateral subclavian and axillary arteries and origins of both common carotid arteries, which remained patent. The celiac, superior mesenteric, and right renal artery were perfused by the true lumen, and a false patent lumen extending into the left renal artery was noticed.

At the level of the aortic bifurcation into common iliac arteries, the true lumen was markedly narrowed, and minimal perfusion noticed into the false lumen. The left common internal 
iliac artery was noted to arise from the true lumen; however, it was minuscule as well, reducing flow significantly to the left lower extremity. There was a monophasic Doppler signal on the left left while and an absent Doppler signal on the right femoral artery. He was hemodynamically stable during the transfer to the operating room.

After general anesthesia and intubation, the left and right CFAs were exposed via a transverse infra-inguinal cut-down. After partial heparinization, a mid-portion of a 10-mm Dacron fabric graft was anastomosed to the left CFA in a side-to-side fashion. This end was connected to the arterial inflow limb of the cardiopulmonary bypass (CBP) machine circuit (Figure1). A clamp was placed on the free end. This graft served to perfuse both the systemic circulation as well as perfuse the left and lower extremity. The other end of the graft was brought through a suprapubic tunnel and sewn to right CFA beyond where the aortic dissection flap had terminated. During this tunneling, sternotomy and caval cannulation, CPB was initiated following full heparinization. Repair of aortic dissection with the replacement of hemi aortic arch using a 32 mm Dacron graft was performed with 18 Celsius of 20 minutes circulatory arrest and retrograde cardioplegia. The aortic valve commissures were suspended as well. After completing the aortic dissection repair, lower extremity anastomosis, and expeditious separation of CPB, we noticed suboptimal perfusion to the right lower extremity despite adequate systemic hemodynamics and cardiac function. We had high suspicion that aortic dissection flap most likely progressed and resulted in significant flow reduction into both CFA.

Another possibility was that the abdominal aorta dissection flap could have now occluded flow to both iliac arteries. The patient was not in a hybrid room to determine exact etiology, nor did we have the technical expertise to perform an acute dissection flap's fenestration. We determined that extra-anatomic arterial perfusion was necessary to perfuse the limbs because of persistent lower extremity malperfusion. An 8-mm ring reinforced Polytetrafluoroethylene

(PTFE) graft was anastomosed to the perfused right axillary artery true lumen and the end to the proximal origin of the right femoral. After protamine reversal, a standard 4-compartment fasciotomy was performed on the index limb to prevent compartment syndrome. The patient's postoperative course was notable for rhabdomyolysis and non-oliguric acute renal failure, which recovered fully without dialysis. The patient recovered without any neurological deficit. He had palpable distal pulses throughout in all four extremities at 2 weeks discharge to rehabilitation. The patient has been followed up for 4 years and remains fully functional, in full-time employment (Figure 2).

\section{Comment}

Rapid reestablishment of flow within the ascending aortic true lumen is paramount in alleviating 
dynamic malperfusion syndromes occurring in up to $90 \%$ who sustain an aortic dissection at the time of aortic repair (1).

However, static and fixed malperfusion can persist despite aortic repair of dissection. If static malperfusion is not managed immediately, it can be deadly regardless of whether the malperfusion is addressed before or after aortic repair and removal of an intimal tear $(2,3)$. We believe that malperfusion should be addressed simultaneously at type A dissection repair with growing advocacy $(4,5)$. We modified our perfusion graft as an alternative technique (figure1) to perfuse both extremities and systemic circulation expeditiously. This allowed immediate reperfusion of the threatened limb during replacement of the ascending thoracic aortic, thereby limiting irreversible limb ischemia.

\section{References}

1. Girdauskas E, Kuntze T, Borger MA, Falk V, Mohr FW. Surgical risk of preoperative malperfusion in acute type A aortic dissection. J Thorac Cardiovasc Surg. 2009;138:1363-9.

2. Geirsson A, Szeto WY, Pochettino A, McGarvey ML, Keane MG, Woo YJ, et al. Significance of malperfusion syndromes prior to contemporary surgical repair for acute type A dissection: outcomes and need for additional revascularizations. Eur J Cardiothorac Surg. 2007;32:255-62.

3. Patel HJ, Williams DM, Dasika NL, Suzuki Y, Deeb GM. Operative delay for peripheral malperfusion syndrome in acute type A aortic dissection: a long-term analysis. J Thorac Cardiovasc Surg. 2008;135:1288-95; discussion 1295-6.

4. Okita Y. The sooner the better? Early strategies of peripheral vascular intervention for patients with acute aortic dissection complicated by organ malperfusion. J Thorac Cardiovasc Surg. 2018;156(2):490-491.

5. Uchida K, Karube N, Kasama K, et al. Early reperfusion strategy improves the outcomes of surgery for type A acute aortic dissection with malperfusion. J Thorac Cardiovasc Surg. 2018;156(2):483-489.

Figure Legends

Figure 1. This is the author's sketch illustrating the $10 \mathrm{~mm}$ Dacron graft (colored red) with cardiopulmonary bypass inflow at A, side to side anastomosis of graft to CFA at (B) and tunneled across (C) to the right CFA (D). The graft was sawn closed at B following separation at bypass.

Figure 2 . CTA with 3 D reconstruction illustrating the extra-anatomical grafts, ascending aortic graft and dissection flap at 3.5 years. 


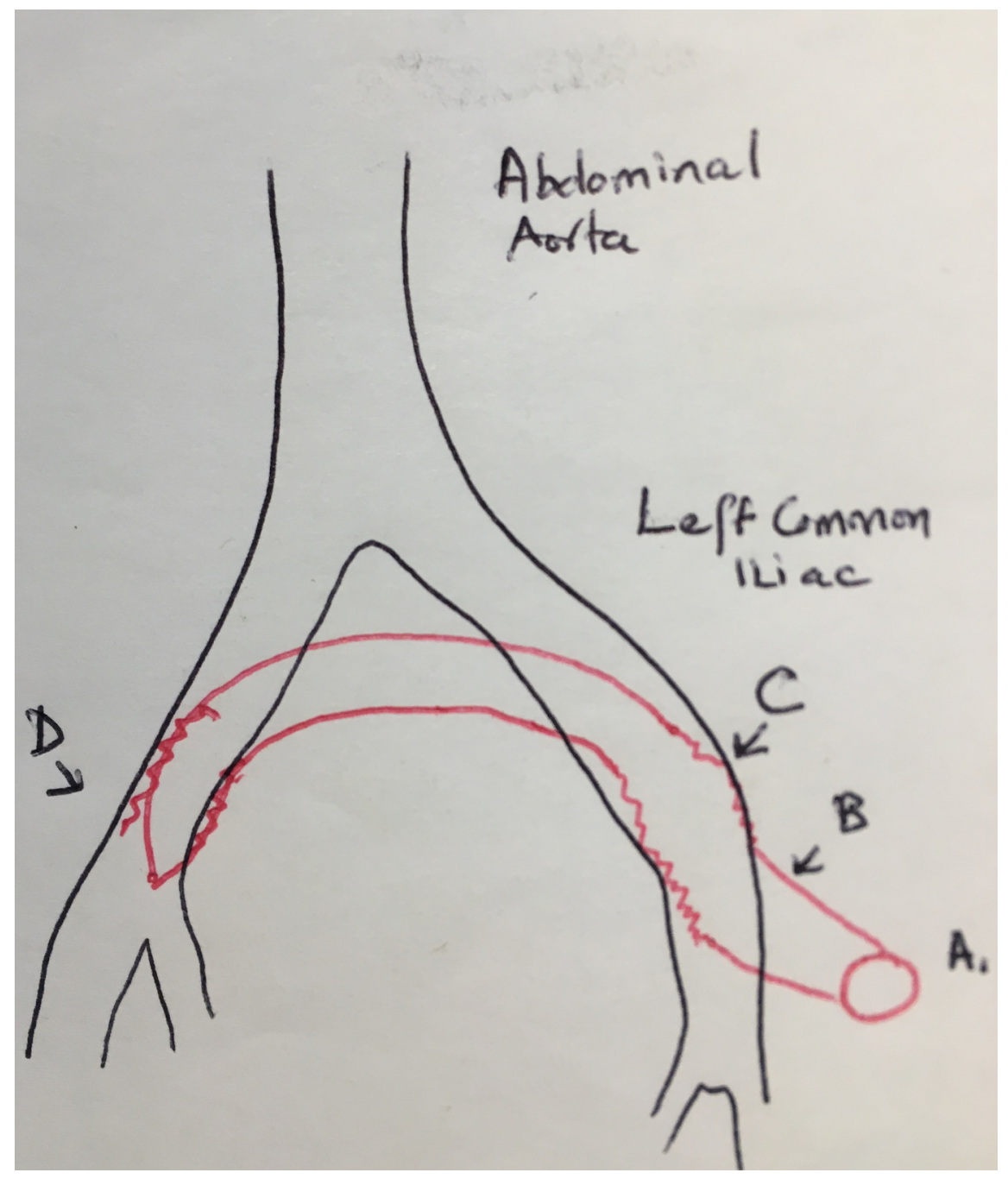




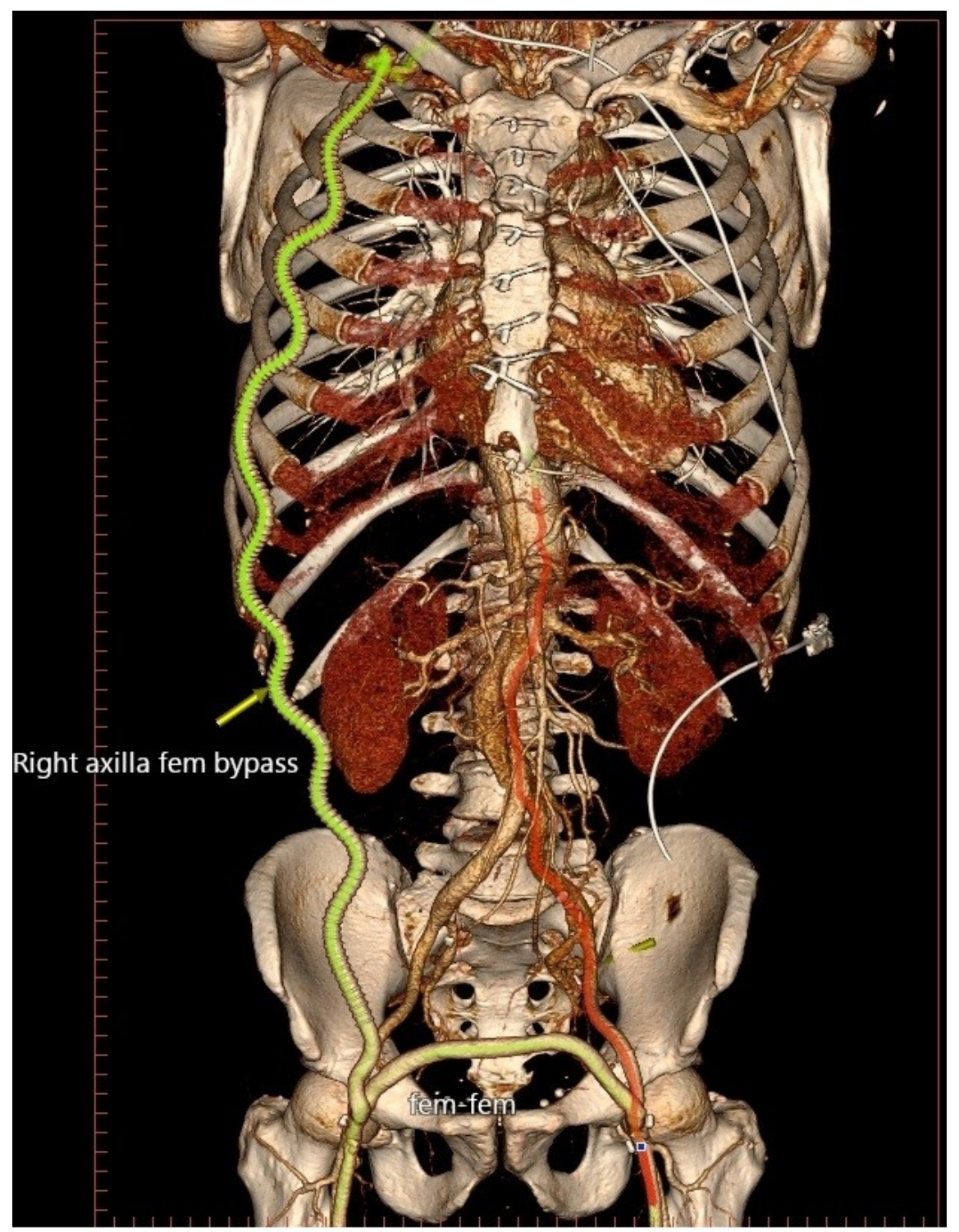

